\title{
KRAS mutational analysis and immunohistochemical studies can help distinguish pancreatic metastases from primary lung adenocarcinomas
}

\author{
Alyssa M Krasinskas, Simon I Chiosea, Timothy Pal and Sanja Dacic \\ Department of Pathology, University of Pittsburgh Medical Center, Pittsburgh, PA, USA
}

\begin{abstract}
Lung metastases from primary pancreatic adenocarcinomas often have mucinous features, which makes them difficult to distinguish from the primary lung adenocarcinoma. We explored the potential utility of KRAS mutational status and immunohistochemical studies in the evaluation of adenocarcinomas in the lungs of patients with known pancreatic cancer. Metastatic pancreatic cancer cases had fewer solitary lung lesions (5 (15\%) versus $37(95 \%)$ for lung primaries; $P=0.0001)$, more tumors with pure $(100 \%)$ mucinous morphology (16 (50\%) versus 9 (23\%) for lung primaries; $P=0.0037)$, and more frequent KRAS mutations (24 (75\%) versus 18 (46\%) for lung primaries; $P=0.0093$ ). Presence of the KRAS G12C mutation had $96 \%$ specificity and positive predictive value for lung adenocarcinoma, whereas G12R was $99 \%$ specific for pancreatic cancer with a positive predictive value of $86 \%$. Of the 18 KRAS mutated mucinous lung tumors, only $3(16 \%)$ occurred in nonsmokers. Conversely, of the 19 KRAS mutated pancreatic cancer metastases, $11(58 \%)$ occurred in nonsmokers. The median overall survival was significantly shorter for patients with metastatic tumors when compared with patients with primary mucinous tumors (19 months, 95\% confidence interval, 10-28 months versus 55 months, 95\% confidence interval, 39-70 months, $P=0.005)$. CK20 and CDX2 positivity supported metastatic pancreatic cancer, whereas TTF-1 positivity supported primary lung adenocarcinoma. In summary, KRAS G12C mutations, TTF-1, and napsin A were associated with primary lung adenocarcinoma, whereas KRAS G12R mutations, CK20, and CDX2 favored pancreatic adenocarcinoma. We showed survival differences for patients whose pancreatic metastases were synchronous versus metachronous to their primary tumors, and for patients with mucinous pancreatic cancer metastases versus primary mucinous lung adenocarcinomas. Differences in KRAS mutations reflect differences in exposure to tobacco smoking and highlight biological differences between two KRAS oncogene-driven cancers.
\end{abstract}

Modern Pathology (2014) 27, 262-270; doi:10.1038/modpathol.2013.146; published online 26 July 2013

Keywords: KRAS; lung cancer; mucinous adenocarcinoma; pancreatic cancer

Patients with pancreatic cancer often develop distant metastases. The most common site of distant metastases is the liver, followed by the peritoneum and the lung. ${ }^{1-4}$ Making the distinction between primary lung adenocarcinoma and pulmonary metastases from the pancreatic ductal adenocarcinoma is often difficult based on histopathological and immunophenotypical features alone. Both lung and pancreatic adenocarcinomas share similar morphology, and immunohistochemical markers,

Correspondence: Dr AM Krasinskas, MD, Department of Pathology, Emory University, 1364 Clifton Road NE, Room H-180, Atlanta, GA 30322, USA.

E-mail: akrasin@emory.edu

Received 22 April 2013; revised 5 June 2013; accepted 5 June 2013; published online 26 July 2013 such as TTF-1 and napsin A for lung adenocarcinoma, cannot always distinguish primary from metastatic disease..$^{5-7}$

Although the clinical diagnosis of metastatic disease is often straightforward based on radiographic evidence of either more than one site of involvement or multiple lesions in a single organ, lung cancer can also produce synchronous lesions. In recent studies, the frequencies of multifocal lung tumors range from 3.7 to $24 \% \cdot{ }^{8-11}$ Hence, the presence of more than one lung lesion cannot be used as the sole discriminator of primary from metastatic disease.

Although pancreatic cancer is usually rapidly fatal with median survival after resection of only 14-20 months ${ }^{12}$ and an overall 5-year survival rate of $6 \%$ for all stages ${ }^{13}$ some patients can have 
a prolonged clinical course with the development of late metastasis. In a recent study of pulmonary resections for isolated pancreatic adenocarcinoma metastases, the median time from pancreatectomy (with no evidence of distant disease) to identification of the first pulmonary nodule on imaging was 29 months and to metastectomy was 34 months. ${ }^{14}$ Hence, when a patient with a known history of pancreatic cancer develops a lung lesion or lesions without other organ involvement, the distinction between metastatic disease and a lung primary can be difficult.

Activating KRAS mutations are present in the majority (about 90\%) of pancreatic ductal adenocarcinomas $^{15-17}$ and less frequently (13-23\%) in lung adenocarcinomas. ${ }^{18-20}$ On the basis of the limitations of clinical and histopathological parameters in distinguishing the primary from metastatic disease, we wished to explore the potential utility of molecular studies, specifically KRAS mutational status, in the evaluation of adenocarcinomas of the lung in patients with known pancreatic cancer, using large cohorts of primary lung adenocarcinomas and pancreatic adenocarcinomas for comparison. We also tested a small panel of immunohistochemical stains on a subset of cases to determine the utility of these markers.

\section{Materials and methods}

\section{Patients}

The slides and reports of all potential pancreatic cancer metastases to the lung from the surgical pathology files at our institution over a 5-year period (2006-2011) were reviewed. All cases that had formalin-fixed paraffin-embedded tissue blocks available for the study were included for a total of 32 cases. Clinicopathologic and follow-up data were recorded.

Three different comparison groups were used. One group $(n=94)$ consisted of a consecutive cohort of primary pancreatic ductal adenocarcinoma cases resected between 2007 and 2009 at UPMC with known KRAS mutation status (previously included in another study). ${ }^{21}$ The second group $(n=39)$ included primary mucinous adenocarcinomas of the lung with available tissue for analysis. ${ }^{18}$ The final group $(n=284)$ included primary adenocarcinomas of the lung that harbored KRAS mutations. ${ }^{22-23}$ This study was approved by the University of Pittsburgh Institutional Review Board (IRB no. PRO11070413).

\section{Immunohistochemistry}

Standard automated immunohistochemistry was performed for CK20, CDX2, TTF-1, napsin A, MUC2, and SMAD4 (Table 1) on formalin-fixed,
Table 1 Antibodies used in this study

\begin{tabular}{llc} 
Antibody & Clone/company & Dilution \\
\hline CDX2 & CDX2-88/BioGenex (Fremont, CA) & $1: 200$ \\
CK20 & Ks20.8/Dako (Carpinteria, CA) & $1: 50$ \\
MUC2 & Ccp58/Vector Laboratories (Burlingame, CA) & $1: 25$ \\
Napsin A & Polyclonal/Cell Marque (Rocklin, CA) & Predilute \\
SMAD4 & B-8/Santa Cruz Biotechnology (Dallas, TX) & $1: 500$ \\
TTF-1 & 8G7G3/1/Dako & $1: 50$ \\
\hline
\end{tabular}

paraffin-embedded, 4- $\mu$ thick tissue sections. Following deparaffinization in xylene and rehydration in ethanol, the slides were stained using the Ventana BenchMark XT; the enzymatic reactivity was visualized with the iVIEW DAB Detection Kit (Ventana Medical Systems, Tucson, AZ).

The immunohistochemical stains were scored as follows: 0 (no positivity or only very occasional cell staining); $1+(5-10 \%$ of cells stained); and $2+$ ( $>10 \%$ of cells stained). The intensity of staining was also scored on a categorical scale from 0 to 2 where 0 indicated absent; $1+$, very weak; and $2+$, definite staining. Only tumor cells that stained in the appropriate nuclear or cytoplasmic location were scored. Nuclear staining for TTF-1 and CDX2, and cytoplasmic staining for CK20, napsin A, and MUC2 were considered positive. For statistical analyses, a score of 0 was considered negative and scores of $1+$ and $2+$ were considered positive. For SMAD4, a score of 0 in both the cytoplasmic and nuclear compartments was considered as lost/ decreased staining.

\section{KRAS Exon 2 Codons 12 and 13 Mutational Analysis}

Exon 2 KRAS codon 12 and 13 mutations were assessed using the Sanger sequencing in both directions (forward and reverse) as previously described. ${ }^{24}$ Tumor areas with more than 50\% tumor cells (based on review of hematoxylin and eosin stained slides) were microdissected from the $4-\mu \mathrm{m}$ unstained formalin-fixed paraffin-embedded sections.

\section{Statistical Analysis}

A $t$-test was used to characterize the relationship between quantitative variables, and the Fisher exact test was used to characterize the relationship between categorical variables. Median survival intervals with 95\% confidence intervals were estimated using the Kaplan-Meier method, with statistical significance of differences between groups estimated using the log-rank test. Patients with 3 months or less survival after the tissue diagnosis were excluded from the survival analysis. A $P$-value of $<0.05$ was defined as statistically significant. Statistical analysis was performed using SPSS 20 (Somers, NY, USA). 
Table 2 Clinicopathological features of pancreatic adenocarcinomas metastatic to lung and lung primaries

\begin{tabular}{|c|c|c|c|c|}
\hline & $\begin{array}{l}\text { (1) PDAC all } \\
\text { metastases }(\mathrm{n}=32)\end{array}$ & $\begin{array}{l}\text { (2) PDAC mucinous } \\
\text { metastases }(\mathrm{n}=27)\end{array}$ & $\begin{array}{l}\text { (3) Primary lung with } \\
\text { mucinous features }(\mathrm{n}=39)\end{array}$ & $\begin{array}{l}\text { P-value } \\
(2 \text { vs } 3)\end{array}$ \\
\hline $\operatorname{Sex}(M / F)$ & $17 / 15$ & $14 / 13$ & $21 / 18$ & NS \\
\hline Age (mean), vears & 64 (range, 48-78) & 64 (range, 48-78) & 69 (range, 50-90) & NS \\
\hline Nonsmoker/ever smoker & $13 / 17$ & $11 / 14$ & $6 / 32$ & 0.02 \\
\hline \multicolumn{5}{|l|}{ Focality } \\
\hline Solitary & 5 & 5 & 37 & 0.0001 \\
\hline Multifocal & 24 & 21 & 2 & \\
\hline \multicolumn{5}{|l|}{ Primary tumor location ${ }^{a}$} \\
\hline Head/neck & 23 & 18 & NA & NA \\
\hline Body/tail & 7 & 7 & NA & \\
\hline \multicolumn{5}{|l|}{ Morphology $\mathrm{b}$} \\
\hline Pure (100\%) MC & 16 & 16 & 9 & 0.0037 \\
\hline$>90 \%$ Mucinous & 16 & 16 & 19 & NS \\
\hline$>50 \%$ Mucinous & 20 & 20 & 33 & NS \\
\hline Nonmucinous ${ }^{\mathrm{C}}$ & 3 & 0 & 0 & NS \\
\hline \multicolumn{5}{|l|}{$K R A S^{\mathrm{d}}$} \\
\hline Mutation & 24 & 20 & 18 & 0.0093 \\
\hline Wild-type & 5 & 5 & 21 & \\
\hline \multicolumn{5}{|l|}{ KRAS mutations $\mathrm{e}^{\mathrm{e}}$} \\
\hline $\mathrm{G} 12 \mathrm{D}(\mathrm{G}>\mathrm{A})$ & 9 & 7 & 3 & NS \\
\hline $\mathrm{G} 12 \mathrm{~V}(\mathrm{G}>\mathrm{T})$ & 7 & 7 & 6 & NS \\
\hline G12R $(\mathrm{G}>\mathrm{C})$ & 5 & 4 & 0 & NS \\
\hline $\mathrm{G} 12 \mathrm{C}(\mathrm{G}>\mathrm{T})$ & 2 & 1 & 7 & 0.019 \\
\hline G12A $(\mathrm{G}>\mathrm{C})$ & 0 & 0 & 1 & NS \\
\hline $\mathrm{G} 12 \mathrm{~F}(\mathrm{G}>\mathrm{T})$ & 0 & 0 & 1 & NS \\
\hline
\end{tabular}

Abbreviations: F, female; M, male; MC, mucinous carcinoma; NA, not applicable, NS, not significant; PDAC, pancreatic ductal adenocarcinoma. a Unknown, $n=2$.

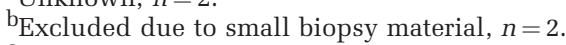

${ }^{\mathrm{C}}$ The morphology of the three nonmucinous tumors was acinar/cribriform, papillary, and solid.

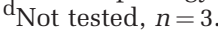

${ }^{\mathrm{e}}$ Unknown, $n=1$.

\section{Results}

Metastatic Pancreatic Adenocarcinomas: Clinicopathological Data, KRAS Mutational Analysis, and Immunohistochemical Profile

The clinicopathological features of the 32 study patients with pancreatic ductal adenocarcinoma metastatic to the lung are shown in Table 2. All but three patients (91\%) had only pulmonary involvement by metastatic disease (two also had liver and one also had omental metastases). One half of the patients $(n=16)$ had metastatic disease at the time the pancreatic primary was diagnosed. The other half had prior pancreatic resections with M0 disease; the average time from pancreatic resection to the lung biopsy or resection was 30 months (range, 4-63 months). Of the five patients with solitary lung metastases, the mean time from pancreatectomy to lung biopsy/resection was 37 months (range, 19-63 months), and the mean overall survival for these 5 patients was 75 months (and three patients are still alive).

KRAS mutations were present in 24 of the 29 $(83 \%)$ metastatic pancreatic adenocarcinomas that were tested. All the mutations involved codon 12 of the KRAS gene. Thirteen of these patients had tissue from their primary pancreatic cancers available for mutational analysis. The presence or absence of KRAS mutations was concordant in $11(85 \%)$ of cases: In two cases, both tumors were wild-type and in eight cases, the specific KRAS mutations were concordant (G12V, $n=4, \mathrm{G} 12 \mathrm{D}, n=2$, and G12R, $n=2$ ); in one case, the pancreatic primary tumor had a G12V mutation, and the metastasis had a G12C mutation; in two cases, the primary tumors were wild-type and the metastases had G12D mutations.

The metastatic pancreatic ductal adenocarcinomas had the following immunohistochemical profile: CK20 + in 17/31 (55\%), CDX2 + in 17/32 $(53 \%)$, MUC2 + in $4 / 29(14 \%)$, TTF-1 + in $1 / 31$ (3\%), napsin $\mathrm{A}+$ in $0 / 29(0 \%)$, and loss of SMAD4 in $12 / 32(38 \%)$.

\section{Comparison of Tumors with Mucinous Features: Metastatic Pancreatic Adenocarcinomas and Primary Lung Adenocarcinomas}

As the majority (90\%) of metastatic pancreatic adenocarcinomas in this study had mucinous features, we performed a head-to-head comparison with a cohort of 39 primary lung adenocarcinomas with mucinous features. ${ }^{18}$ The clinicopathological 

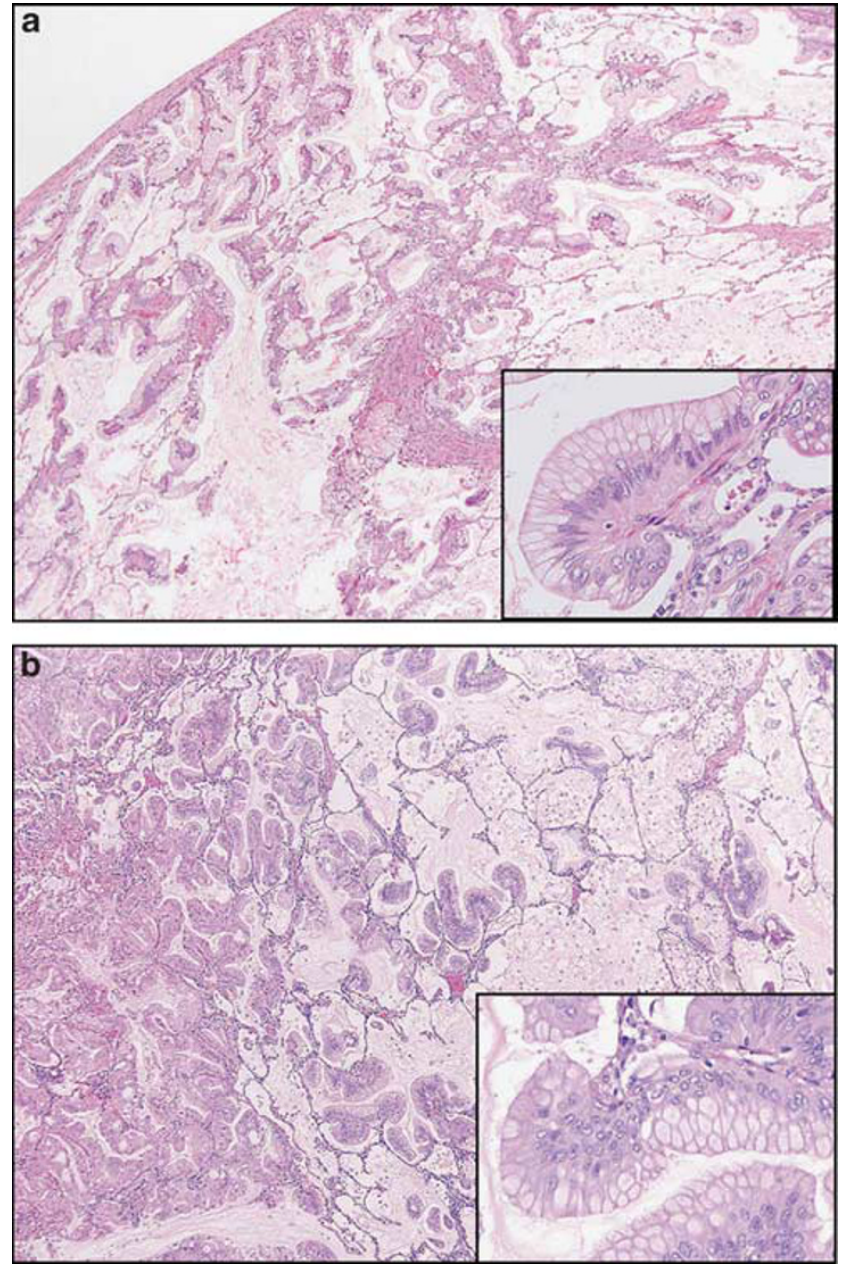

Figure 1 (a) Pancreatic ductal adenocarcinoma metastatic to the lung (hematoxylin and eosin stain, $\times 40$; inset; $\times 100$ ) closely resembles (b) primary mucinous lung adenocarcinoma (hematoxylin and eosin stain, $\times 40$; inset; $\times 100$ ).

data for these two groups are also shown in Table 2, and representative histological appearances of metastatic and primary mucinous adenocarcinomas are shown in Figure 1. Gender and mean age were similar between the two groups, but there were significant differences in other parameters. In the mucinous pancreatic cancer metastases cohort, there were fewer smokers or ever smokers $(P=0.02)$, many more multiple (versus solitary) lesions $(P=0.0001)$, and more tumors with pure $(100 \%)$ mucinous morphology $(P=0.0037)$.

KRAS mutations were more frequent in mucinous pancreatic cancer metastases compared with the mucinous lung primaries $(P=0.0093)$ (Table 2). Of interest, the glycine to cysteine (G12C) transversion was uncommon in the pancreatic cancer metastases, but was the most commonly encountered mutation in lung primaries $(P=0.019)$. Although the numbers are small, it is interesting that only one of the five $(20 \%)$ metastatic pancreatic tumors that produced a solitary lesion harbored a KRAS mutation.

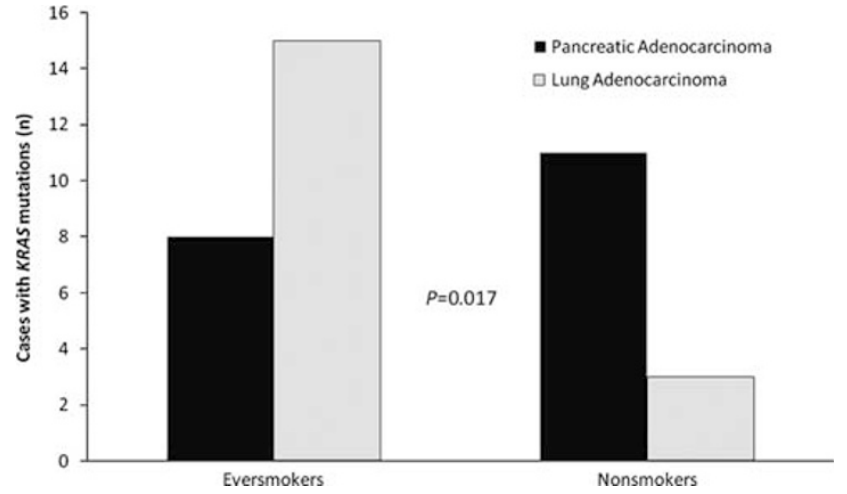

Figure 2 Comparison of the number of cases of KRAS mutated metastatic pancreatic adenocarcinoma and KRAS mutated primary mucinous lung adenocarcinoma in ever smokers and nonsmokers. KRAS mutations were more common in ever smokers with mucinous lung adenocarcinomas and in nonsmokers with pancreatic adenocarcinoma $(P=0.017)$.

Table 3 Sensitivity, specificity, positive, and negative predictive values for pancreatic and lung adenocarcinomas

\begin{tabular}{|c|c|c|c|c|}
\hline & $\begin{array}{l}\text { Sensitivity } \\
(\%)\end{array}$ & $\begin{array}{l}\text { Specificity } \\
(\%)\end{array}$ & $\begin{array}{l}P P V \\
(\%)\end{array}$ & $\begin{array}{l}N P V \\
(\%)\end{array}$ \\
\hline \multicolumn{5}{|c|}{ Mucious PDAC metastases } \\
\hline KRAS mutation & 80 & 54 & 53 & 81 \\
\hline CK20 + & 65 & 72 & 77 & 59 \\
\hline CDX2 + & 63 & 94 & 94 & 63 \\
\hline \multicolumn{5}{|c|}{ Mucinous lung primaries } \\
\hline KRAS G12C & 39 & 95 & 88 & 62 \\
\hline TTF-1 + & 76 & 96 & 94 & 83 \\
\hline Napsin A + & 33 & 100 & 100 & 81 \\
\hline \multicolumn{5}{|c|}{$\begin{array}{l}\text { KRAS mutated lung } \\
\text { primaries }\end{array}$} \\
\hline KRAS G12C & 44 & 96 & 96 & 41 \\
\hline \multicolumn{5}{|c|}{ KRAS mutated PDAC } \\
\hline KRAS G12R & 15 & 99 & 86 & 74 \\
\hline
\end{tabular}

Abbreviations: NPV, negative predictive value; PDAC, pancreatic ductal adenocarcinoma; PPV, positive predictive value.

Smoking history was assessed in patients with primary and secondary mucinous tumors. KRAS mutations were found in 15/32 (47\%) of ever smokers and in $3 / 6(50 \%)$ of nonsmokers with primary mucinous lung cancer, and in 8/11 (72\%) of ever smokers and in 11/11 (100\%) of nonsmokers with pancreatic cancer metastases, but this did not reach statistical significance $(P=0.21)$. However, when only KRAS mutated tumors were assessed, $15 / 18(84 \%)$ occurred in ever smokers and only $3 / 18$ $(16 \%)$ occurred in nonsmokers with primary mucinous lung cancer, whereas 8/19 (42\%) occurred in ever smokers and 11/19 (58\%) occurred in nonsmokers with mucinous pancreatic cancer metastases. The association of smoking history with KRAS mutation status was significantly different between pancreatic metastases and lung primaries $(P=0.017$; Figure 2). Interestingly, the G12C mutation $(n=8)$ 

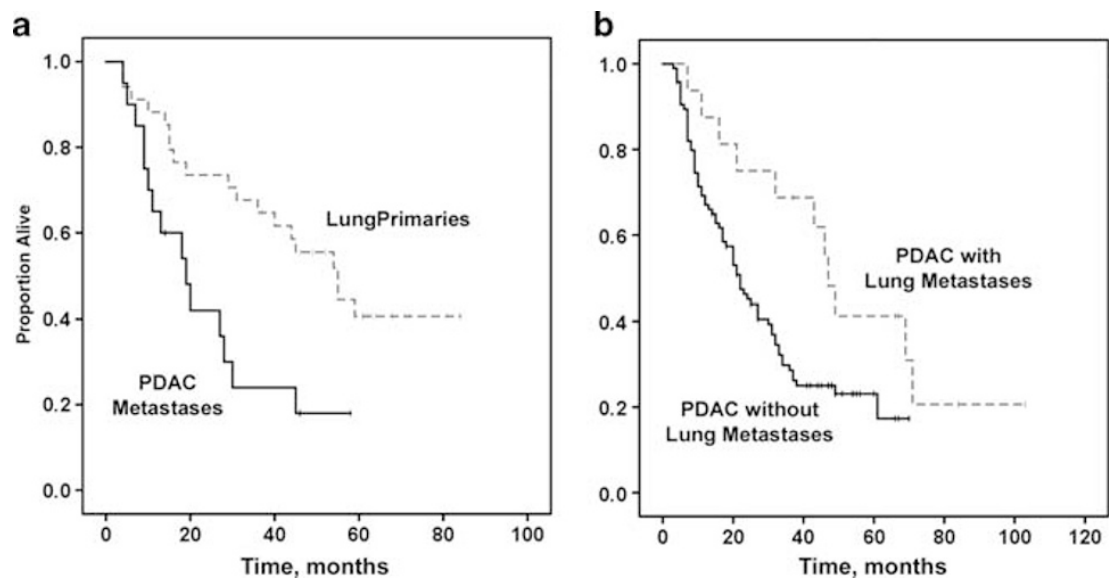

Figure 3 Kaplan-Meier curves showing overall survival in (a) patients with metastatic pancreatic adenocarcinoma to the lung (all with mucinous features) compared with patients with primary mucinous lung adenocarcinoma, and (b) patients with pancreatic adenocarcinoma with or without lung-only metastases.

Table 4 Summary of immunohistochemical staining patterns in tumors with mucinous features

\begin{tabular}{lccc}
\hline & $\begin{array}{c}\text { Pancreatic metastases } \\
\text { (\% positive) }\end{array}$ & $\begin{array}{c}\text { Lung primaries } \\
\text { (\% positive) }\end{array}$ & P-value \\
\hline CK20 & $17 / 26(65)$ & $5 / 18(28)$ & 0.03 \\
CDX2 & $17 / 27(63)$ & $1 / 18(6)$ & 0.0001 \\
MUC2 & $4 / 24(17)$ & $1 / 10(10)$ & NS \\
TTF-1 & $1 / 26(4)$ & $16 / 21(76)$ & 0.0001 \\
Napsin A & $0 / 25(0)$ & $3 / 9(33)$ & 0.014 \\
SMAD4 loss & $10 / 27(37)$ & $8 / 28(28)$ & NS \\
(\%loss) & & & \\
\hline
\end{tabular}

Abbreviation: NS, not significant.

was only seen in smokers $(P=0.015)$. The $\mathrm{G}>\mathrm{T}$ transversion was more common in smokers $(n=17$ versus $n=5$ in nonsmokers), whereas the $G>A$ transition and $\mathrm{G}>\mathrm{C}$ transversion were more common in nonsmokers $(n=6$ and 3 , respectively, in nonsmokers versus $n=4$ and 2 , respectively, for smokers $)(P=0.058)$.

The detection of a KRAS mutation as a marker of metastatic pancreatic carcinoma (versus primary lung adenocarcinoma) has a sensitivity of $80 \%$, but a specificity of only $54 \%$ and positive predictive value of $53 \%$ (Table 3 ). However, if a G12C mutation is used to support a lung primary, while the sensitivity is low (39\%), the specificity is high (95\%) and the positive predictive value is $87.5 \%$.

For the tumors with mucinous features, the average follow-up was 16 months from lung tissue diagnosis for the metastatic pancreatic tumors compared with an average follow-up of 38 months for the lung primaries. The median overall survival was significantly shorter for patients with metastatic tumors when compared with overall survival of patients with primary mucinous tumors (19 months, $95 \%$ confidence interval, 10-28 months versus 55 months, 95\% confidence interval, 39-70 months, $P=0.005$ ) (Figure 3a). Although there was a trend toward worse overall survival for patients with KRAS mutated tumors, this did not reach statistical significance $(P=0.07$; data not shown).

The immunohistochemical profiles were different between lung primary and metastatic pancreatic tumors (Table 4). CK20 and CDX2 positivity supports metastatic pancreatic cancer, whereas TTF-1 positivity supports primary lung adenocarcinoma. Although napsin A expression favors primary lung adenocarcinoma, only $33 \%$ of the cases were positive in this series. The sensitivities, specificities, positive predictive values, and negative predictive values for these immunostains in adenocarcinomas with mucinous features are shown in Table 3.

\section{Comparison of Tumors with KRAS Mutations: Metastatic Pancreatic, Primary Pancreatic, and Primary Lung Adenocarcinomas}

As the majority ( $83 \%$ ) of pancreatic adenocarcinoma metastases harbored KRAS mutations, we next performed head-to-head comparisons of KRAS mutations in metastases with a cohort of 284 KRAS mutated primary lung adenocarcinomas and a cohort of KRAS mutated pancreatic ductal adenocarcinoma primary resections. Of note, this cohort of primary lung tumors included the 18 KRAS mutated mucinous tumors described above and is shown in Table 2. Although there were no differences in mean age between the three groups, there was a difference in gender (Table 5). Of the primary pancreatic cancers with KRAS mutations, $55 \%$ were in men, whereas of the primary lung cancers with KRAS mutations, only $41 \%$ were in men $(P=0.032)$.

There were several differences in the types of KRAS mutations that were encountered in primary lung adenocarcinomas compared with both primary and metastatic pancreatic adenocarcinomas. The four most common amino-acid substitutions (KRAS 
Table 5 KRAS mutated adenocarcinomas (pancreatic metastases and primaries, and lung primaries)

\begin{tabular}{|c|c|c|c|c|}
\hline & $\begin{array}{l}\text { (1) Metastatic PDAC } \\
K R A S+\left(\mathrm{n}=23^{\mathrm{a}}\right)\end{array}$ & $\begin{array}{l}\text { (2) Primary PDAC } \\
K R A S+(\mathrm{n}=94)\end{array}$ & $\begin{array}{l}\text { (3) Primary lung } \\
K R A S+(\mathrm{n}=284)\end{array}$ & $\mathrm{P}$-value \\
\hline $\operatorname{Sex}(M / F)$ & $13 / 10$ & $53 / 42$ & $117 / 167$ & 0.032 (2 vs 3) \\
\hline Age (mean), years & 63 (range, 48-77) & 67 (range, 32-88) & 66 (range, 39-92) & NS \\
\hline \multicolumn{5}{|c|}{ KRAS mutations (amino acid/nucleotide) } \\
\hline \multicolumn{5}{|l|}{ Transition mutations } \\
\hline G12D/GGT $\rightarrow$ GAT & 9 & 50 & 45 & 0.009 (1 vs 3), 0.0001 (2 vs 3 ) \\
\hline G12S/GGT $\rightarrow$ AGT & 0 & 0 & 9 & NS \\
\hline Total & 9 & 50 & 54 & $<0.0001(2 \text { vs } 3)^{\mathrm{b}}$ \\
\hline \multicolumn{5}{|l|}{ Transversion mutations } \\
\hline G12V/GGT $\rightarrow$ GTT & 7 & 27 & 53 & NS (1 vs 3), 0.042 (2 vs 3) \\
\hline G12R/GGT $\rightarrow$ CGT & 5 & 13 & 3 & 0.0001 ( 1 or 2 vs 3$)$ \\
\hline G12C/GGT $\rightarrow$ TGT & 2 & 3 & 125 & 0.0007 (1 vs 3), 0.0001 (2 vs 3$)$ \\
\hline G12A/GGT $\rightarrow$ GCT & 0 & 1 & 22 & NS (1 vs 3),0.022 (2 vs 3) \\
\hline $\mathrm{G} 12 \mathrm{~F} / \mathrm{GGT} \rightarrow \mathrm{TTT}$ & 0 & 0 & 7 & NS \\
\hline G12L/GGT $\rightarrow$ CTT & 0 & 0 & 1 & NS \\
\hline Total & 14 & 44 & 211 & $<0.0001(2 \text { vs } 3)^{\mathrm{b}}$ \\
\hline \multicolumn{5}{|l|}{ Codon 13 mutations } \\
\hline G13x & 0 & 0 & 19 & NS \\
\hline
\end{tabular}

Abbreviations: AA, amino acid; NS, not significant; PDAC, pancreatic ductal adenocarcinoma; $\mathrm{x}$ is either D, C, or V.

${ }^{a} A$ total of 24 metastatic PDAC harbored KRAS mutations, but the specific mutation was unknown in one case, so this case was excluded from this table.

$\mathrm{b}_{P}<0.0001$ for transition versus transversion mutations in primary pancreatic versus primary lung adenocarcinomas.

mutations) in pancreatic cancers in decreasing frequency were: G12D, G12V, G12R, and G12C; the four most common amino-acid substitutions in primary lung adenocarcinomas in decreasing frequency were: G12C, G12V, G12D, and G12A (all of these amino-acid substitutions were statistically significant between the groups; see Table 5 and Figure 4a). Several amino-acid changes were only noted in lung adenocarcinomas, including G12F, G12L, G12S, and codon 13 mutations $(P=$ NS due to small numbers), and only one pancreatic tumor (primary) had a G12A mutation $(P=0.022)$. The most frequent nucleotide change in primary lung tumors was $\mathrm{G}>\mathrm{T}(n=185)$, followed by $\mathrm{G}>\mathrm{A}$ $(n=54)$ and $\mathrm{G}>\mathrm{C} \quad(n=26)$ compared with the nucleotide changes in the metastatic pancreatic cancer of $\mathrm{G}>\mathrm{T}(n=8), \mathrm{G}>\mathrm{A} \quad(n=7)$, and $\mathrm{G}>\mathrm{C}$ $(n=4) ; P=0.036$. As shown in Table 5, when KRAS mutations were compared as either transition or transversion mutation, there were significantly more transversion mutations in lung adenocarcinomas (211 transversions and 54 transitions) than pancreatic adenocarcinomas (44 transversions and 50 transitions; $P<0.0001$ ) (Figure $4 \mathrm{~b}$ ).

The detection of specific KRAS mutations can help to predict the pancreatic versus lung adenocarcinoma (Table 3). The detection of G12C has a high specificity and a positive predictive value for lung adenocarcinoma (96\% for both), whereas G12R is highly specific (99\%) for pancreatic cancer with a high PPV of $86 \%$. Although G12D is the most common mutation in pancreatic cancer and the third most common in lung adenocarcinoma, it has low sensitivity, moderate specificity, and low PPV of $50 \%, 84 \%$ and $57 \%$, respectively.

\section{Pancreatic Adenocarcinomas with and without Lung Metastases}

The survival of all patients who underwent pancreatic adenocarcinoma resections, some of whom later developed lung metastases, was assessed. Those patients who later had their lung-only metastases biopsied or resected had significantly longer survival (from the time of pancreatectomy) compared with the control cohort of 94 patients with pancreatic cancer resections (all without lung biopsies). The median survival for those patients with lung metastases (single or multiple) was 47 months (95\% confidence interval, 42-52 months) versus 22 months (95\% confidence interval, 17-27 months) for the cohort of resected pancreatic cancers $(P=0.028)$ (Figure $3 b$ ).

\section{Discussion}

When patients with pancreatic cancer either present with or later develop lung-only lesions, the tissue is often obtained for a confirmation of the metastatic disease. However, distinguishing primary adenocarcinomas of the lung from the metastatic disease can be challenging, especially when the pancreatic ductal adenocarcinoma is a consideration. When pancreatic cancers metastasize to the lung, they often have mucinous features and can mimic primary mucinous adenocarcinomas of the lung. ${ }^{25}$

Studies have evaluated the utility of immunohistochemical markers in distinguishing lung primaries from the metastatic disease ${ }^{26-30}$ or determining the site of unknown primaries. ${ }^{31}$ Studies such as these 


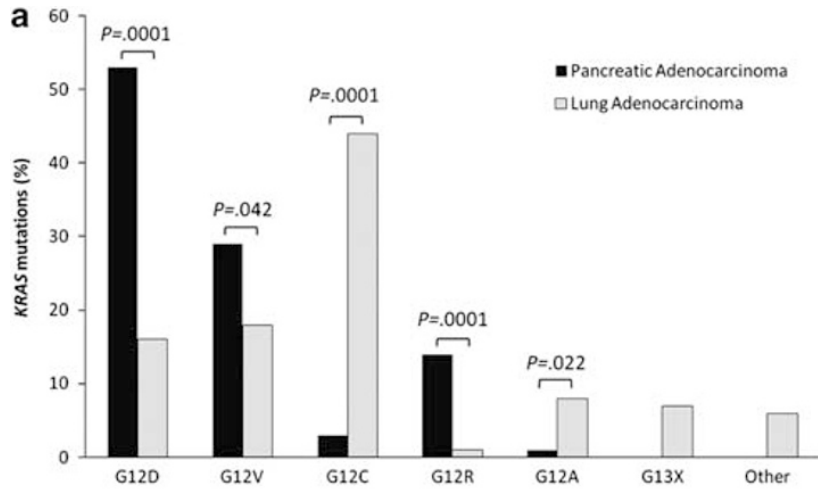

b

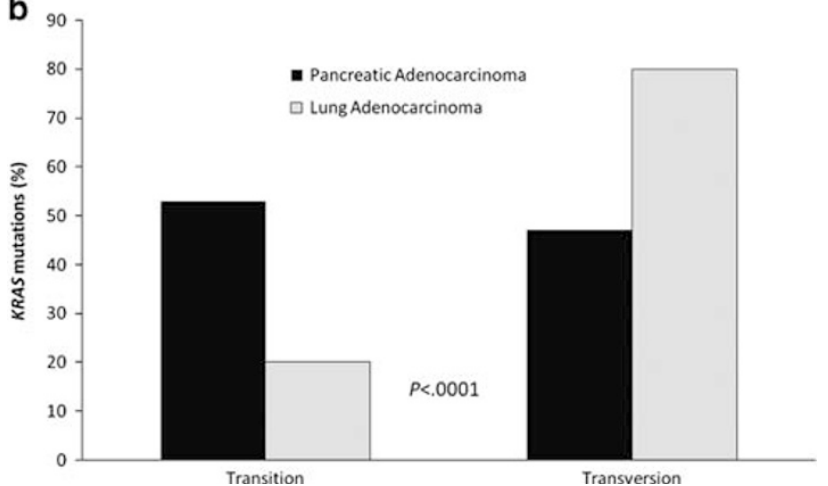

Figure 4 KRAS mutation types observed in primary pancreatic adenocarcinomas and primary lung adenocarcinomas. (a) Aminoacid mutations (b) KRAS transition (substitution of a purine for a purine or a pyrimidine for a pyrimidine) and transversion (substitution of a pyrimidine for a purine or purine for a pyrimidine) mutations.

have shown that TTF-1 and napsin A are specific markers for primary lung adenocarcinoma. Few studies, however, have explored the utility of these markers in distinguishing mucinous metastases from mucinous lung primaries. It appears that mucinous tumors are less frequently positive for napsin A $(0-33 \%)$ and TTF-1 $(0-42 \%) .{ }^{6-7}$ In our immunohistochemical evaluation of tumors with mucinous features, only $33 \%$ of lung primaries expressed napsin A, and when focal staining was counted as positive, $76 \%$ of lung tumors expressed TTF-1. Although statistically different than the expression seen in pancreatic cancer metastases $(P=0.014$ and 0.0001 , respectively), the positivity rate is rather low. Similarly, although CK20 and CDX2 positivity in pancreatic cancer metastases were both statistically different than in lung primaries $(P=0.03$ and 0.0001 , respectively), only $63-65 \%$ of cases were positive. Hence, we feel that the utility of these immunohistochemical markers may have limited diagnostic utility for distinguishing primary lung from metastatic pancreatic adenocarcinomas with mucinous features.

Because of the limitations of immunohistochemistry, we sought to determine whether molecular findings could help distinguish lung primaries from pancreatic cancer metastases. Similar to the literature, KRAS mutations were more frequent in mucinous pancreatic cancer metastases compared with the mucinous lung primaries (80 versus $54 \%$, 0.0093). By comparing the KRAS mutation status of four different cohorts of tumors, we were able to determine that particular KRAS mutations can help predict the presence of either a lung primary or metastatic pancreatic cancer. The detection of G12C has a high specificity and a positive predictive value for lung adenocarcinoma ( $96 \%$ for both), whereas G12R is highly specific (99\%) for pancreatic cancer with a high positive predictive value of $86 \%$. The high frequency of the G12C transversion in mucinous lung cancer (followed by G12V and G12D) has also been shown previously. ${ }^{32}$

PCR based clonality assays can be used to distinguish synchronous or metachronous primaries from the metastatic disease when both tumors in question have adequate tissue for molecular analysis. ${ }^{33}$ However, clonality assays can have a $3-17 \%$ non-informative rate and, for loss of heterozygosity assays, there is a need to analyze normal tissue. ${ }^{11,33-34}$ The comparison of KRAS mutation profiles can be used to confirm pancreatic metastases to the lung, as demonstrated in our study and in a prior case report. ${ }^{35}$ In our study, there was an overall concordance rate of $77 \%$.

Making the distinction between pancreatic cancer metastases and a primary lung cancer is not only useful for the clinical management of these patients but it can also have an impact on prognosis. In our cohort of mucinous tumors, patients with a diagnosis of metastatic pancreatic adenocarcinoma, many of whom presented with metastatic disease at the time of diagnosis, had a significantly shorter overall survival than did patients with primary lung adenocarcinomas (19 months versus 55 months, $P=0.005$ ). However, when only patients who had prior pancreatectomies for M0 pancreatic cancer who subsequently developed lung metastases were studied, those patients had significantly longer survival (from the time of pancreatectomy) compared with the control cohort of patients with pancreatic cancer resections without lung-only metastases (47 months versus 22 months, respectively, $P=0.028$ ). These findings are supported by a recent study that showed improved survival of patients with isolated lung metastasis from pancreatic adenocarcinoma who underwent pulmonary metastectomy. ${ }^{14}$ These observations suggest that pancreatic cancers that metastasize late and to only the lung may have a different and more indolent biology than those that metastasize to the lung early or are present at the time of pancreatic cancer diagnosis.

One additional interesting observation was the association between KRAS mutation status and smoking in metastatic pancreatic cancer. Pancreatic cancer metastases harbored KRAS mutations in $72 \%$ of ever smokers and $100 \%$ of nonsmokers. This is in contrast to primary lung adenocarcinoma in which KRAS mutations are highly associated with smoking 
and are uncommonly found in nonsmokers. ${ }^{18,36-39}$ However, similar to our findings, other studies of pancreatic cancer have found no difference in KRAS mutation frequencies in smokers and nonsmokers. ${ }^{40-41}$

Although the frequency of KRAS mutations in ever smokers in our mucinous lung cancer cohort $(47 \%)$ was similar to other reports, ${ }^{36,38}$ the frequency of $50 \%$ for KRAS mutations in nonsmokers is very high, as most studies report frequencies in lung cancer between 2 and 15\%. ${ }^{18,36-39}$ The high frequency in our study could be related to the small sample size or to the fact these were mucinous lung primaries. One study that specifically correlated smoking history with the mucinous phenotype found that mucinous adenocarcinoma had little relationship to smoking history. ${ }^{19}$ As previously noted in the literature, G12C transversions are induced by the carcinogens in tobacco smoke and in our study were found in ever smokers only. ${ }^{32,36}$

In summary, although it is often difficult to distinguish primary adenocarcinomas of the lung from metastatic pancreatic adenocarcinoma, we have shown that the immunohistochemical assessment of TTF-1, napsin A, CDX2, and CK20 together with KRAS mutational analysis may have diagnostic utility in some cases. We also showed that there are survival differences for patients whose pancreatic metastases are synchronous versus metachronous to their primary tumors and for patients with pancreatic cancer metastases versus primary lung adenocarcinomas. Finally, we highlighted potential biological differences between pancreatic cancer and a subset of lung adenocarcinomas. Although the KRAS oncogene has a critical role in the carcinogenesis of these two tumors, when studied deeper, there are biological differences, including specific types of mutations encountered in each tumor and the association (or non-association) with smoking.

\section{Acknowledgements}

We thank members of the personnel in the Molecular Anatomic Pathology Laboratories at UPMC for their excellent technical support. Support for advice regarding regulatory matters was provided by the National Institutes of Health through Grant Numbers UL1 RR024153 and UL1TR000005.

\section{Disclosure/conflict of interest}

The authors declare no conflict of interest.

\section{References}

1 Iacobuzio-Donahue CA, Fu B, Yachida S, et al. DPC4 gene status of the primary carcinoma correlates with patterns of failure in patients with pancreatic cancer. J Clin Oncol 2009;27:1806-1813.

2 Kamisawa T, Isawa T, Koike M, et al. Hematogenous metastases of pancreatic ductal carcinoma. Pancreas 1995;11:345-349.

3 Mao C, Domenico DR, Kim K, et al. Observations on the developmental patterns and the consequences of pancreatic exocrine adenocarcinoma. Findings of 154 autopsies. Arch Surg 1995;130:125-134.

4 Yachida S, Iacobuzio-Donahue CA. The pathology and genetics of metastatic pancreatic cancer. Arch Pathol Lab Med 2009;133:413-422.

5 Mukhopadhyay S. Utility of small biopsies for diagnosis of lung nodules: doing more with less. Mod Pathol 2012;25(Suppl 1):S43-S57.

6 Chu PG, Chung L, Weiss LM, et al. Determining the site of origin of mucinous adenocarcinoma: an immunohistochemical study of 175 cases. Am J Surg Pathol 2011;35:1830-1836.

$7 \mathrm{Wu}$ J, Chu PG, Jiang Z, et al. Napsin A expression in primary mucin-producing adenocarcinomas of the lung: an immunohistochemical study. Am J Clin Pathol 2013;139:160-166.

8 Flieder DB, Vazquez M, Carter D, et al. Pathologic findings of lung tumors diagnosed on baseline CT screening. Am J Surg Pathol 2006;30:606-613.

9 Nakata M, Sawada S, Yamashita M, et al. Surgical treatments for multiple primary adenocarcinoma of the lung. Ann Thorac Surg 2004;78:1194-1199.

10 Rostad H, Strand TE, Naalsund A, et al. Resected synchronous primary malignant lung tumors: a population-based study. Ann Thorac Surg 2008;85:204-209.

11 Takamochi K, Oh S, Matsuoka J, et al. Clonality status of multifocal lung adenocarcinomas based on the mutation patterns of EGFR and K-ras. Lung Cancer 2012;75:313-320.

12 Bachmann J, Michalski CW, Martignoni ME, et al. Pancreatic resection for pancreatic cancer. HPB (Oxford) 2006;8:346-351.

13 American cancer society. Cancer facts \& figures. Accessed January 5 2013; Available from http://www. cancer.org/Research/CancerFactsFigures/index.

14 Arnaoutakis GJ, Rangachari D, Laheru DA, et al. Pulmonary resection for isolated pancreatic adenocarcinoma metastasis: an analysis of outcomes and survival. J Gastrointest Surg 2011;15:1611-1617.

15 Almoguera C, Shibata D, Forrester K, et al. Most human carcinomas of the exocrine pancreas contain mutant c-K-ras genes. Cell 1988;53:549-554.

16 Smit VT, Boot AJ, Smits AM, et al. KRAS codon 12 mutations occur very frequently in pancreatic adenocarcinomas. Nucleic Acids Res 1988;16:7773-7782.

17 Hruban RH, van Mansfeld AD, Offerhaus GJ, et al. K-ras oncogene activation in adenocarcinoma of the human pancreas. A study of 82 carcinomas using a combination of mutant-enriched polymerase chain reaction analysis and allele-specific oligonucleotide hybridization. Am J Pathol 1993;143:545-554.

18 Dacic S, Shuai Y, Yousem S, et al. Clinicopathological predictors of EGFR/KRAS mutational status in primary lung adenocarcinomas. Mod Pathol 2010;23:159-168.

19 Kakegawa S, Shimizu K, Sugano M, et al. Clinicopathological features of lung adenocarcinoma with KRAS mutations. Cancer 2011;117:4257-4266.

20 Yoshizawa A, Sumiyoshi S, Sonobe M, et al. Validation of the IASLC/ATS/ERS lung adenocarcinoma classification for prognosis and association with EGFR and 
KRAS gene mutations: analysis of 440 Japanese patients. J Thorac Oncol 2013;8:52-61.

21 Krasinskas AM, Moser AJ, Saka B, et al. KRAS mutant allele specific imbalance is associated with worse prognosis in pancreatic cancer and progression to undifferentiated carcinoma of the pancreas. Mod Pathol 2013 (in press).

22 Chiosea SI, Sherer CK, Jelic T, et al. KRAS mutant allele-specific imbalance in lung adenocarcinoma. Mod Pathol 2011;24:1571-1577.

23 Villaruz LC, Socinski MA, Cunningham DE, et al. The prognostic and predictive value of KRAS oncogene substitutions in lung adenocarcinoma. Cancer 2013; 119:2268-2274.

24 Chiosea S, Shuai Y, Cieply K, et al. EGFR fluorescence in situ hybridization-positive lung adenocarcinoma: incidence of coexisting KRAS and BRAF mutations. Hum Pathol 2010;41:1053-1060.

25 Gaeta M, Volta S, Scribano E, et al. Air-space pattern in lung metastasis from adenocarcinoma of the GI tract. J Comput Assist Tomogr 1996;20:300-304.

26 Jagirdar J. Application of immunohistochemistry to the diagnosis of primary and metastatic carcinoma to the lung. Arch Pathol Lab Med 2008;132:384-396.

27 Stoll LM, Johnson MW, Gabrielson E, et al. The utility of napsin-A in the identification of primary and metastatic lung adenocarcinoma among cytologically poorly differentiated carcinomas. Cancer Cytopathol 2010;118:441-449.

28 Ye J, Findeis-Hosey JJ, Yang Q, et al. Combination of napsin A and TTF-1 immunohistochemistry helps in differentiating primary lung adenocarcinoma from metastatic carcinoma in the lung. Appl Immunohistochem Mol Morphol 2011;19:313-317.

29 Bishop JA, Sharma R, Illei PB, et al. and thyroid transcription factor-1 expression in carcinomas of the lung, breast, pancreas, colon, kidney, thyroid, and malignant mesothelioma. Hum Pathol 2010;41:20-25.

30 Turner BM, Cagle PT, Sainz IM, et al. Napsin A, a new marker for lung adenocarcinoma, is complementary and more sensitive and specific than thyroid transcription factor 1 in the differential diagnosis of primary pulmonary carcinoma: evaluation of 1674 cases by tissue microarray. Arch Pathol Lab Med 2012;136:163-171.
31 Hashimoto K, Sasajima Y, Ando M, et al. Immunohistochemical profile for unknown primary adenocarcinoma. PLoS One 2012;7:e31181.

32 Marchetti A, Buttitta F, Pellegrini S, et al. Bronchioloalveolar lung carcinomas: K-ras mutations are constant events in the mucinous subtype. J Pathol 1996;179:254-259.

33 Tang M, Pires Y, Schultz M, et al. Microsatellite analysis of synchronous and metachronous tumors: a tool for double primary tumor and metastasis assessment. Diagn Mol Pathol 2003;12:151-159.

34 Warth A, Macher-Goeppinger S, Muley $\mathrm{T}$, et al. Clonality of multifocal nonsmall cell lung cancer: implications for staging and therapy. Eur Respir J 2012;39:1437-1442.

35 Alkhasawneh AN, Dong HJ, Liu C, et al. The role of KRAS mutational analysis to determine the site of origin of metastatic carcinoma to the lung: a case report. Case Rep Pathol 2012;2012:425967.

36 Dogan S, Shen R, Ang DC, et al. Molecular epidemiology of EGFR and KRAS mutations in 3,026 lung adenocarcinomas: higher susceptibility of women to smoking-related KRAS-mutant cancers. Clin Cancer Res 2012;18:6169-6177.

37 Johnson ML, Sima CS, Chaft J, et al. Association of KRAS and EGFR mutations with survival in patients with advanced lung adenocarcinomas. Cancer 2013;119:356-362.

38 Riely GJ, Kris MG, Rosenbaum D, et al. Frequency and distinctive spectrum of KRAS mutations in never smokers with lung adenocarcinoma. Clin Cancer Res 2008;14:5731-5734.

39 Zhang Y, Sun Y, Pan Y, et al. Frequency of driver mutations in lung adenocarcinoma from female neversmokers varies with histologic subtypes and age at diagnosis. Clin Cancer Res 2012;18:1947-1953.

40 Blackford A, Parmigiani G, Kensler TW, et al. Genetic mutations associated with cigarette smoking in pancreatic cancer. Cancer Res 2009;69:3681-3688.

41 Schultz NA, Roslind A, Christensen IJ, et al. Frequencies and prognostic role of KRAS and BRAF mutations in patients with localized pancreatic and ampullary adenocarcinomas. Pancreas 2012;41: 759-766. 